AIAA 2002-3759

\title{
Five Segment Booster (FSB) Abort to Orbit (ATO) Studies
}

Mark Tobias and Donald R. Sauvageau

ATK Thiokol Propulsion

Brigham City, UT

Mark Hines and Norman L. Geiser

Boeing Human Space Flight \& Exploration

Huntington Beach, CA

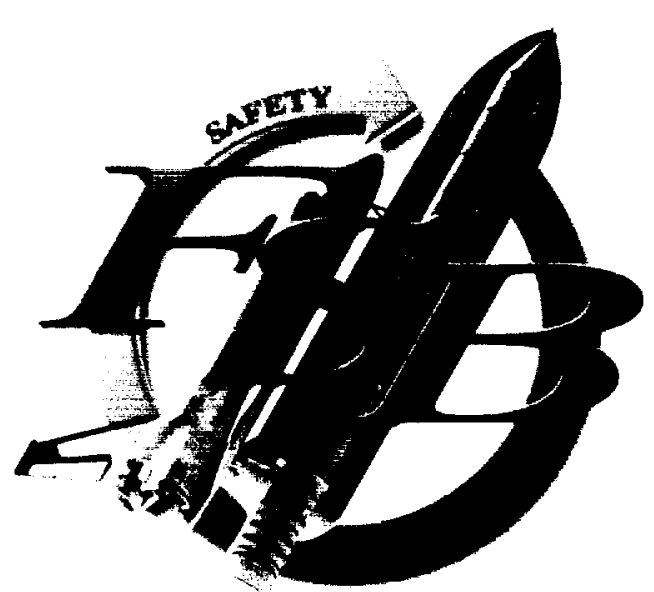

FIVE SEGMENT BOOSTER

\section{AIAA Joint Propulsion Conference July 8-10, 2001 Indianapolis, Indiana}




\title{
FIVE SEGMENT BOOSTER (FSB) ABORT TO ORBIT (ATO) STUDIES
}

\author{
Mark Tobias and Donald R. Sauvageau \\ ATK Thiokol Propulsion \\ Brigham City, UT \\ Mark Hines and Norman L. Geiser \\ Boeing Human Space Flight \& Exploration \\ Huntington Beach, CA
}

The Five Segment Booster ( $:$ SB) concept has been evolving for a number of years as a means to enhance the overall safety and reliability of the Space Shuttle system by minimizing the need to fly the more challenging Return to Launch Site (RTLS) and Transoceanic Abort Landing (TAL) abort profiles. The initial evaluation of the FSB concept was conducted in 1996 to determine the feasibility of the FSB in achieving transatlantic abort landing (TAL) from the pad, thus eliminating the return to launch sitc (RTLS) abort mode. The initial study was conducted by ATK Thiokol and did show the potential for the FSB to eliminate the RTLS abort mode. Later Rockwell (now Boeing) conducted a similar study utilizing FSB performance characteristics and verified that the FSB could indeed achieve TAL from the pad thereby eliminating the necessity for the RTLS abort.

$s$ a result of the potential benelit provided by the FSB, Congress provided NASA moncy to initiate a Phase A feasibility study to assess and mature the basic FSB design approach. In this Phase A feasibility study, all of the major Shuttle elements (Orbiter. External Tank, Booster, Launch and Landing) were involved in assessing the potential implications of the FSB on each of their components. Again, the primary emphasis of the Phase A study was to assess the feasibility of the FSB in eliminating RTLS by achieving TAL from the pad. Another key aspect of the Phase A study was to assess the development cost to qualify a FSB and what the schedule associated with that qualification would be. The Phase A study did confirm the feasibility of developing a FSB with minimal and manageable impacts on other Shuttle elements. It also showed that the FSB enabled the Shutlle to achieve TAL from the pad, thus eliminating RTLS. As a result of the Phase A study, some trajectory enhancements werc identified that would be acceptable for an abort mode scenario. With those trajectory enhancements, there was a limited capability to achieve abort to orbit from the pad with the FSBs. The Phase A study also ended up showing the development costs would be approximately $\$ 1.1 \mathrm{~B}$ and the development program would take approximately five years.

As a result of the potential afforded by the FSB as shown in the Phase A study, Boeing and ATK Thiokol Propulsion committed to expending some of their discretionary resources to mature the FSB concept to enhance its ability to achieve ATO from the launch pad. The purpose of this paper is to discuss the details of the enhancements achieved through the internally funded study conducted by Boeing and ATK Thiokol. To better understand the enhancements that were addressed as part of this follow-on study, some background on what was achieved in the Phase A study is appropriate. The basic FSB configuration is shown in Figure 1. Notice the primary aspect of the FSB is the addition of a new center segment to provide the additional impulse. As a

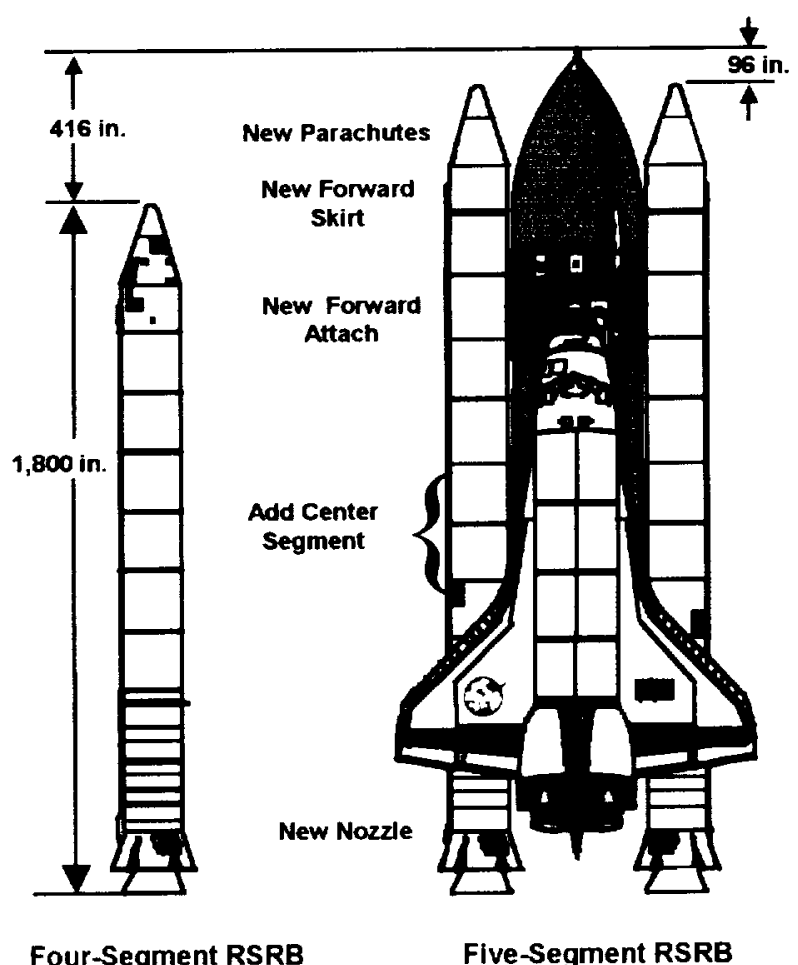

Figure 1. Five-Segment Booster (FSB) Configuration 
result of increasing the total impulse, it became necessary to design a new no:zle to ensure that the pressure capability of the current case hardware was maintained, as well as providing the necessary increase in thrust to meet mission needs. This new nozzle had a larger throat diameter to accommodate the increased mass flow rate associated with adding a center segment. By adding a center segment, the forward attach location to the external tank (ET) is now on the external surface of the forward motor cylinder, as opposed to the previous condition where the ET was attached to the forward skirt. Since the forward skirt no longer needs to transmit the loads from the SRB to the ET, a new forward skint was designed that is a much lighter weight, simpler configuration. As a result of adding an additional center segment, the inert weight of the boosters after separation has increased. Therefore, to maintain the same impact velocity of the booster when it enters the ocean, a new larger diameter parachute was designed. Some of the details of these design changes are shown in Figure 2. Notice the forward motor segment details of the attach are similar to the stiffener segments currently used on the aft segment, which are used to counteract the cavity collapse load after water impact. These stiffeners on the forward segment have a mechanism to allow attaching a thrust post that interfaces with the thrust bolt for the ET. To achieve the desired thrust profile to match the system constraints and accommodate the increased performance capability of the FSB, the forward grain design, inhibitor heights and burn rate had to be changed. Notice the configuration now has 12 fins that are somewhat longer and deeper than the current 11-fin design on the RSRM.

The implications of incorporating a FSB into the Shuttle system relative to the intact abort modes are shown in Figure 3. This figure shows the abort mode opportunities when one SSME goes out at any given time from launch. The time axis shows the total elapsed time from launch when a single SSME is turned off. The abort capability of the Shuttle with the current RSRMs is shown in the blue bar. All conditions are for launches with the International Space Station (ISS) as the destination orbit. With the existing system you can initiate an RTLS at any time between lift off and approximately 250 seconds. The earliest opportunity for a TAL abort occurs at approximately 120 seconds. This means the only abort mode available for the first 120 seconds is an RTLS with the current RSRM boosters. With the current system the ability to achieve an abort to orbit (ATO) becomes available at approximately 250 seconds. The green bars show the enhanced abort capability available when a FSB is substituted for the current RSRM. Notice that the RTLS condition remains essentially the same but the TAL abort opportunity is available from the pad with an SSME throttle setting of 109 percent. By having the ability to initiate a TAL abort from the pad the necessity for an RTLS abort mode is eliminated.
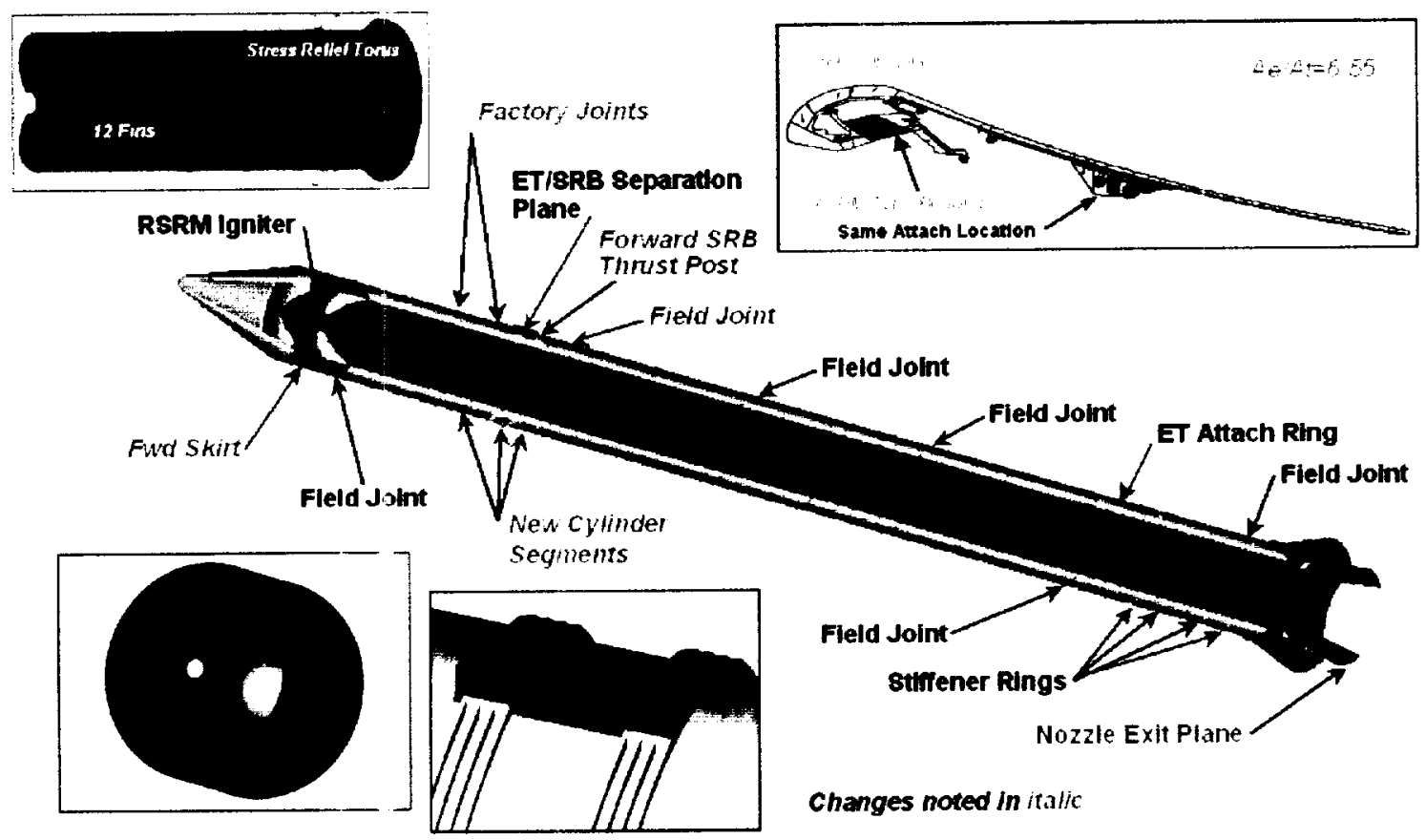

Figure 2. FSB Design Characteristics

Fleld Joint
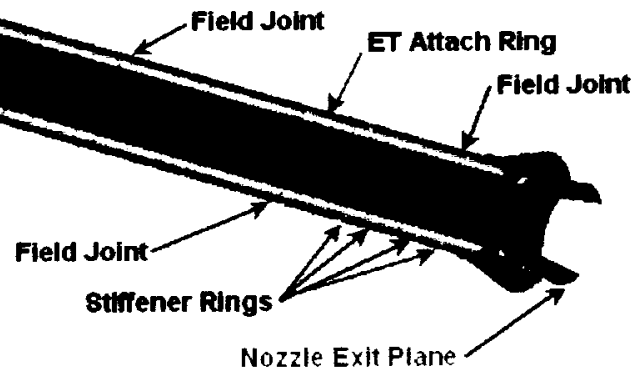

Changes noted in italic 


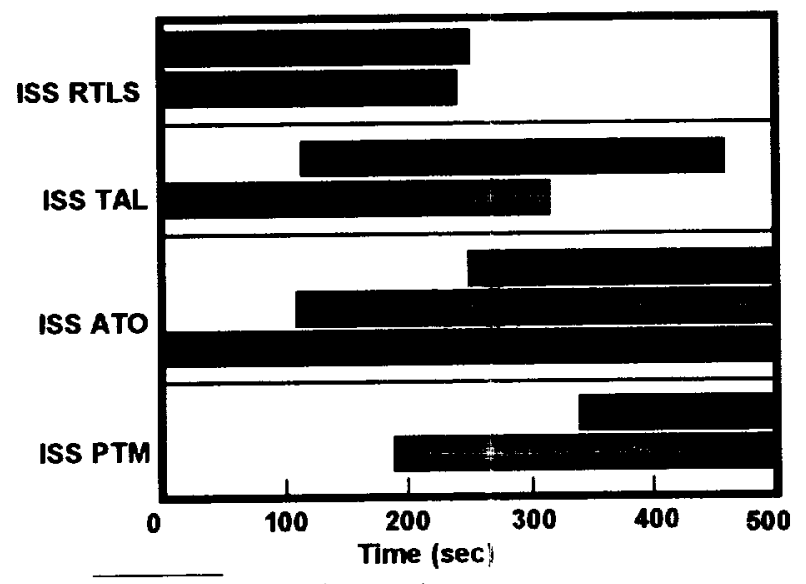

-Apply abort trajectory enhancements

RSRM (SSME 106\%)

Five-Seyment (SSME 104\% - 109\%) Five-Seyment" (SSME 110-112\%)

Figure 3. Shuttle Abort Enhancements With FSB

The capability shown in the green bars and blue bars both utilize the current trajectory constraints. As we proceeded through the Phase A study, a number of trajectory enhancements were :dentified that could be applicable for improving abort capabilities. Many of these enhancements are available due to the extra performance capability afforded by the FSBs. A summary of the potential trajectory enhancements that could be implemented for abort scenarios is shown in Figure 4. Some of the key enhancements include off loading liquid LOX and LH2 lrom the External Tank.
Off loading propellant is possible because there is excess no fail performance when flying with FSBs; therefore. for the nominal missions, you do not need the full load of LOX and LH2, and by eliminating the extra weight associated with the LOX/LH2, abort capability can be greatly enhanced by improving thrust to weight conditions right after booster separation. Additionally, a varicty of trajectory design features called abort enhancements have been identified that can improve abort capability, especially for ISS missions. These enhancements are highlighted in Figure 4 in the text box titled Abort Enhancement Trajectory Design. One critical design feature has to do with steering the vehicle more Easterly upon loss of one SSME to benefit from the Earth's rotation. Additionally, the apogee altitude constraint for recovery of the boosters is relaxed for the abort conditions. This is reasonable because the recovery of the orbiter is more important than the potential for increased attrition rate on the booster hardware when you have an abort situation. By incorporating these trajectory enhancements, the thrust level necessary for the Space Shuttle main engines (SSME) to achieve TAL can be reduced from 109 percent to 104 percent, as a conclusion from Phase A. From recent studies, the purple bar shows that you can achieve ATO from the pad with FSB and an SSME throttle setting of between 110 and 112 percent.

The abort capabilities shown for the FSB configuration utilized the same performance margin that is currently used on the Shuttle RSRM configuration. This may be somewhat conservative relative to incorporating a

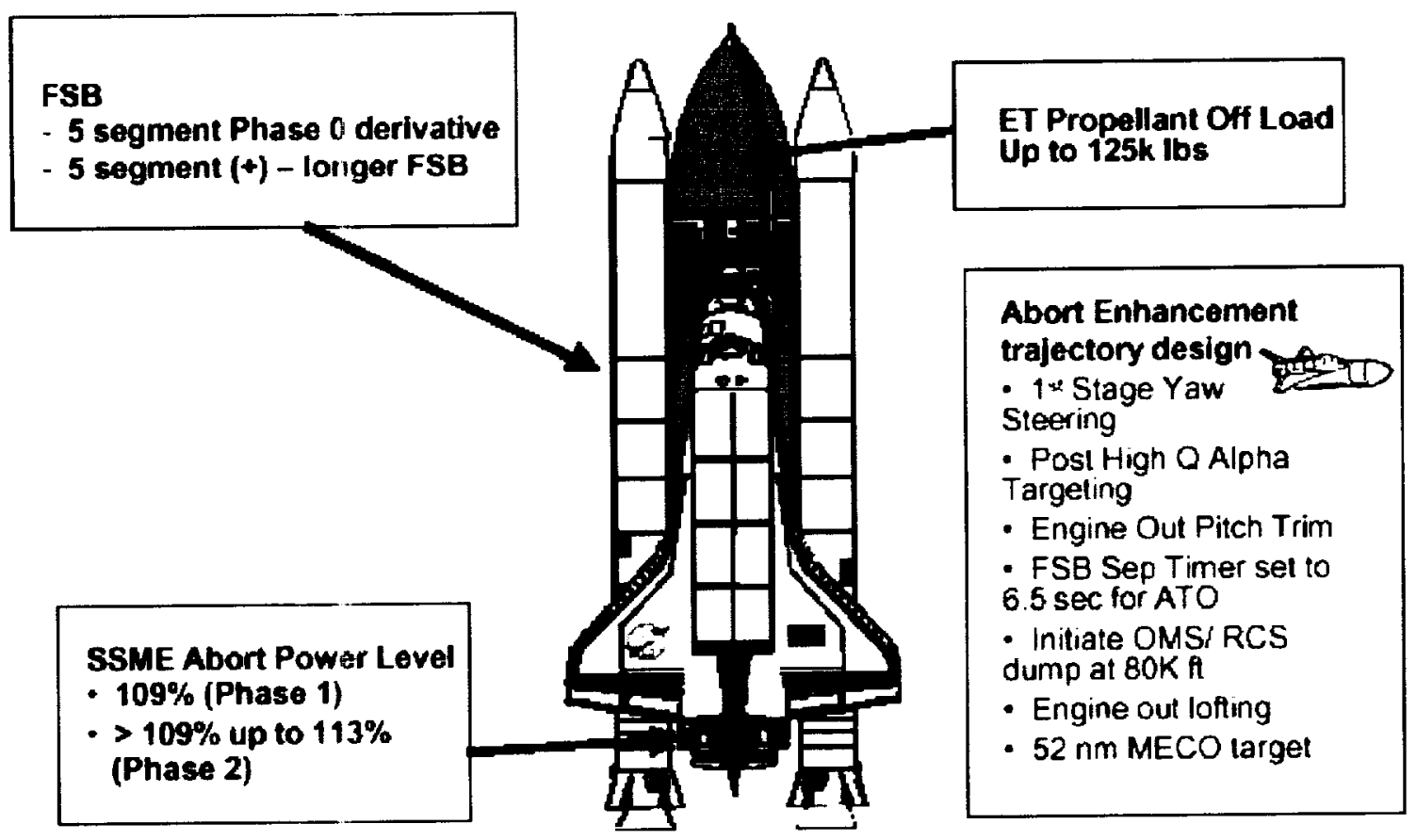

Figure 4. FSB Trajectory Enhancements 
fundamental change to the booster performance characteristics. As part of the internal study conducted by Boeing and ATK Thiokol. it was determined it would be more appropriate to increase the performance margin to reflect potential degradations in performance as the FSB and other system implications mature through the development process. A summary of the performance protection considerations to take those maturation effects into account is summarized in Table 1. This indicates it would te appropriate to have additional performance protection on the order of 7,000 to $9,600 \mathrm{lb}$ over and above the current Shuttle performance margins.

\section{Table 1. ATO Performance Protection Considerations}

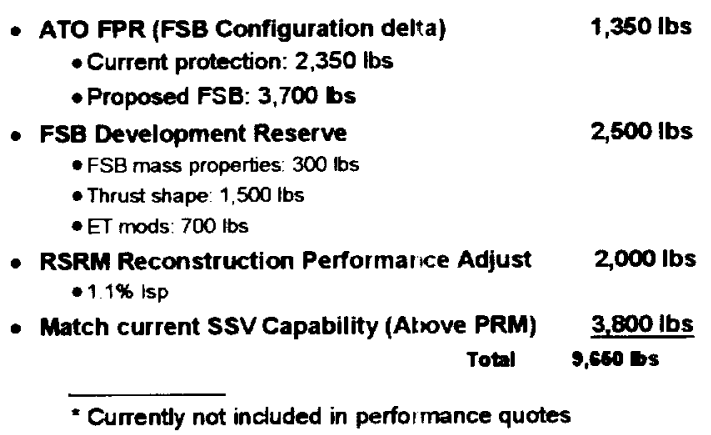

In order to better define the purformance necessary to achieve the desired performance margin, Boeing developed trajectory and performance optimization tools that allowed booster thrust profiles to be optimized to maximizc the performance capability of a given booster configuration while adhering to existing Space Shuttle flight environment constraints (Maximum dynamic pressure, Total load factor, load indicators (ET and Orbiter attach), and thermal indicators. Utilizing the optimization tool developed by Boeing, the optimum thrust profile for the FSB configuration utilized in the Phase A study is shown as the FSB1 thrust trace in Figure 5. Figure 6 shows that when optimum thrust trace is utilized a performance: margin of $1.300 \mathrm{lb}$ is

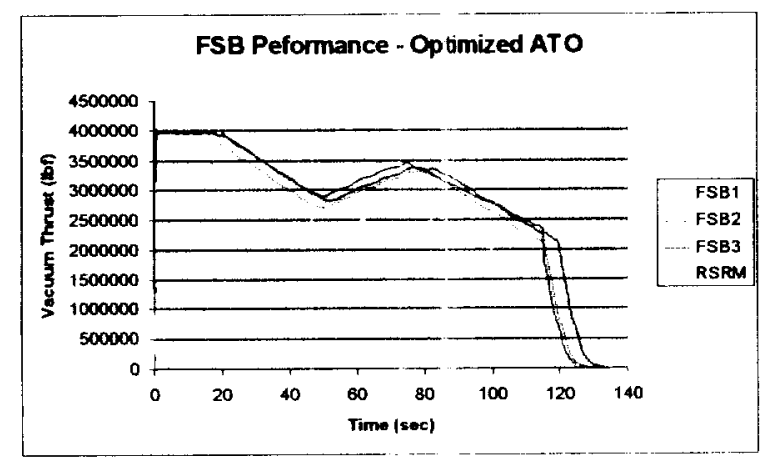

Figure 5. Optimized FSB Thrust Profiles

\begin{tabular}{|c|c|c|c|}
\hline & \multicolumn{2}{|l|}{ Case } & Perform ance \\
\hline Actual Design (Thiokol) & FSB 1031402 & \multicolumn{2}{|r|}{548} \\
\hline Idealized Reference (Boeing) & \multirow[t]{2}{*}{ FSB 1} & \multicolumn{2}{|r|}{1319} \\
\hline \multicolumn{2}{|l|}{ Perform ance Delta } & & -773 \\
\hline \multicolumn{4}{|c|}{ Perform ance Delta B reakdown (Thiokol vs. Boeing) } \\
\hline & Change & Partial & Perform ance Delt a \\
\hline 1 isp (sec) & -0.24 & 993 & -238 \\
\hline Propellant (for a pair) & 1582 & 0.0484 & 77 \\
\hline Drop Wt (tor a pair) & 1598 & -0.125 & -200 \\
\hline Thrust Shape & & & -411 \\
\hline Total & & & .773 \\
\hline
\end{tabular}

Summary

- The Thiokol thrust shape is very similar to the Boeing motor thrust shape

- Sep time is $1.22 \mathrm{sec}$ later for the Thiokol motor

- Perf losses for Thiokol case due to lower Isp, Increased drop wt and tailoff

- Clears all preliminary checks on: LoadsI Thermall Dynamic Pressure/ Max Acceleration

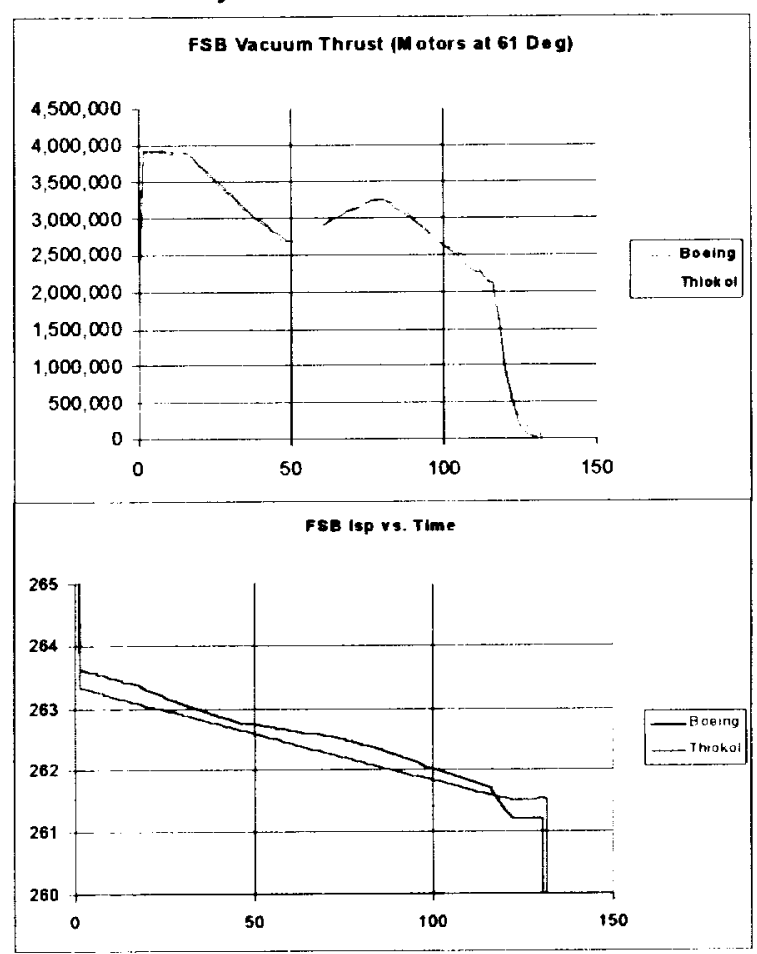

Figure 6. FSB1 Motor Development Summary

achicvable with this FSB in conjunction with a SSME abort throttle setting of 109 percent, abort enhancements, and $118,000 \mathrm{lb}$ propellant off load. The lower thrust trace in Figure 6 shows a comparison of the idealized thrust time profile provided from the Boeing optimization tool, compared to an actual grain design developed by ATK Thiokol. Notice that there is an excellent correlation between the actual performance characteristics and the optimum performance characteristics. When the actual characteristics are into the Boeing trajectory simulation, a performance margin of $550 \mathrm{lb}$ is achieved, so there is a reasonably 
good correlation between optimum and actual performance capability. However, this particular configuration is still well short of the desired $9,000 \mathrm{lb}$ of performance margin goal. To help us come closer to achieving the desired performance goal while maintaining an SSME throttle setting of 109 percent, we extended the length of the FSB an additional 65 in. beyond the configuration evaluated in Phase A. This optimum thrust profile is shown as FSB2 in Figure 5. The optimum performance capability for the FSB2 stretched configuration is shown in Figure 7. Notice that by increasing the length of the FSB an additional 65 in., the performance margin is improved to

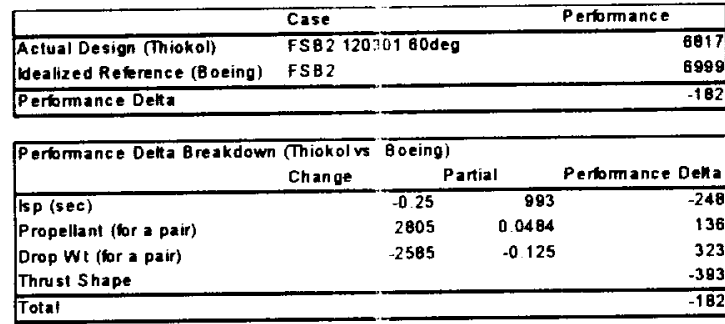

- Summary

- Sep time is $\mathbf{1 . 4 4} \mathrm{sec}$ later for the Thiokol motor

- Increase in prop and decrease in drop weight (along with slight progressive thrust in first 20 sec) help offset Isp and lailoff losses

- Clears all prelim inary checks on: Loads/ Thermal Dynamic Pressure

- Max Acceleration is $3.018 \mathrm{~g}$ 's for July High Energy case with dispersons @ $104 \mathrm{sec}$

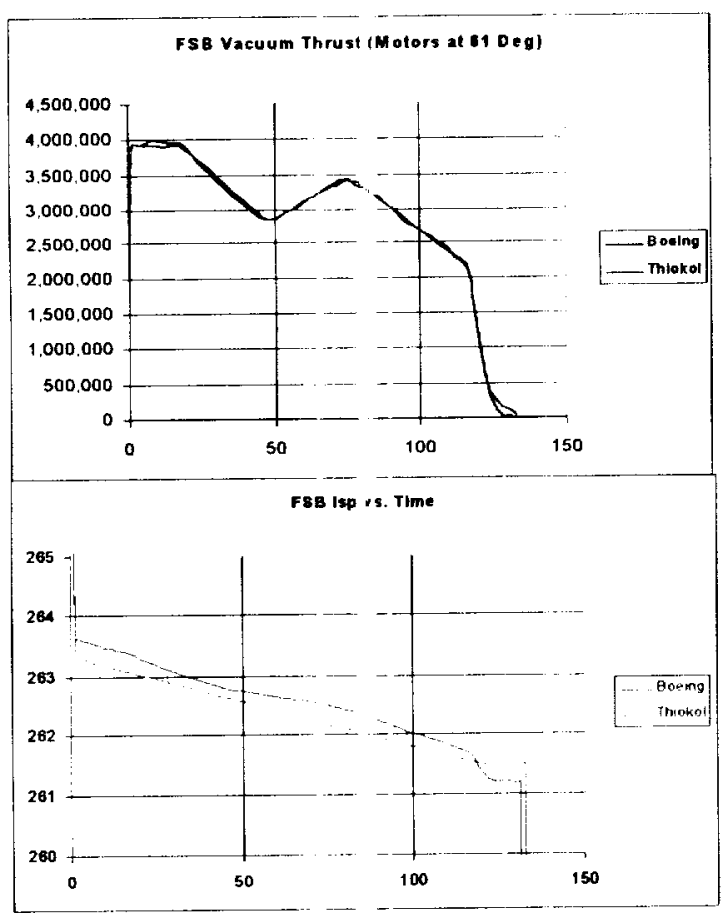

Figure 7. 65 Motor Development Summary approximately $7,000 \mathrm{lb}$. Again, there is a good correlation between the ideal thrust and the actual thrust characteristics from the grain design developed by ATK Thiokol. Since the 65 in. stretch is still short of the desired $9,000 \mathrm{lb}$ performance margin, an additional increase in length was evaluated to determine if additional performance was available from the FSB. FSB-3, shown in Figure 8, is the maximum length that was evaluated as part of the internal study. This increased length was 96 in., which corresponds with a booster length where the tip of the nose cone would be co-planer with the tip of the ET. In this particular configuration, you will notice in Figure 8 the

\begin{tabular}{|c|c|c|c|}
\hline & \multirow{2}{*}{\multicolumn{2}{|c|}{$\begin{array}{l}\text { Case } \\
\text { FSB396b }\end{array}$}} & Perform ance \\
\hline Actual Design (Thiok oi) & & & 8537 \\
\hline Loealized Reference (Boeing) & FSB2 & & 8999 \\
\hline Performance Della & & & 2530 \\
\hline \multicolumn{4}{|c|}{ Performance Detta Breakdawn (Thiok ol vs Boeing) } \\
\hline & Change & Partial & Perform ance Delta \\
\hline $\operatorname{lsp}(\sec )$ & -0.24 & 993 & -238 \\
\hline Propellant (for a pair) & 52773 & 0.0484 & 2.554 \\
\hline $\begin{array}{l}\text { Drop Wt (for a pair) } \\
\text { Thrust Shape }\end{array}$ & 3543 & -0.125 & $\begin{array}{r}-443 \\
865 \\
\end{array}$ \\
\hline Tatal & & & 2.538 \\
\hline
\end{tabular}

- Summary

- Thiokol FSB2 - 96 case causes FTB 5/6 FSB/ ET Attach loads violations from about 72 sec. to 82 sec. Downward slope needs to be started sooner

- Max dynamic pressure violations (10 psf) appear in high energy case from 30 sec. to 42 sec. Most likely caused by progressive thrust not ramping into downward slope soon enough

- Max acceleration violations (3.10 g's) also appear for high energy case at 104 sec. A reduction in thrust starting about $94 \mathrm{sec}$. and continued until tailoff would likely solve this

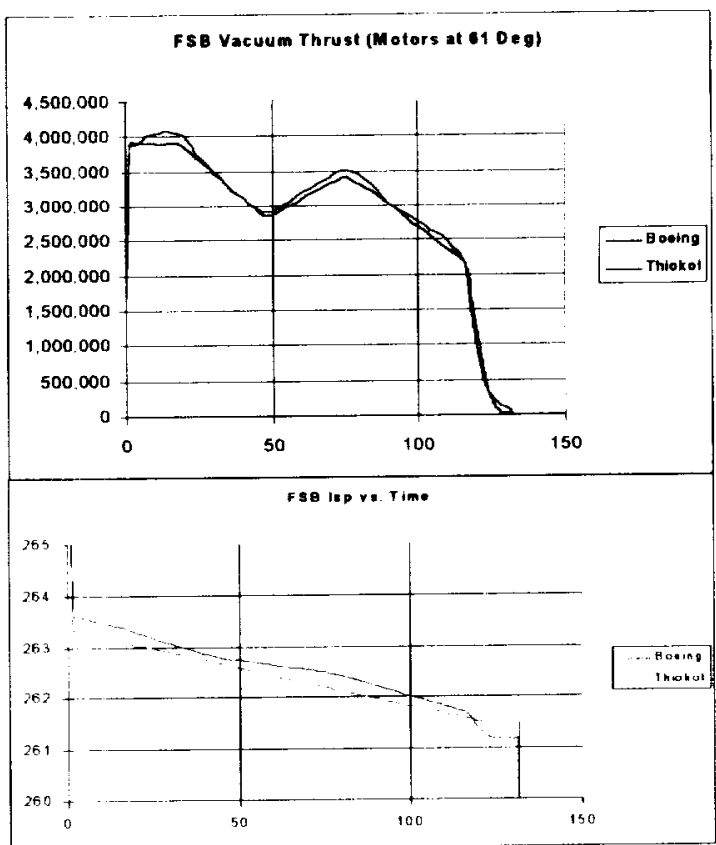

Figure 8. FSB2-96 Motor Development Summary 
performance margin was incrcased to approximately $9,500 \mathrm{lb}$. This is consistent with the desired performance goal of approximately $9,600 \mathrm{lb}$. There are some issues associated with this increased length having to do with dynamic pressure and maximum acceleration considerations both of which can be mitigated through additional design refinement, which will be done in future studies.

As mentioned earlier, one of the key aspects of achieving ATO from the launch pad (lift off) is offloading propellant from the ET. This effect is shown in Figure 9. Notice that the top two curves show the effect of propellant off load in the nominal no fail mission scenario. Because of the enhanced capability afforded by the FSB, one can off load propellant from the ET up to approximately $200,000 \mathrm{lb}$ and still achieve the desired performance capability, which corresponds with a zero propellant margin condition. However, if you observe the bottom two curves for abort scenarios, the abort capability increases as propellant is off loaded. The lower abort curve shows thi condition for the Phase A FSB configuration with the optimized thrust profile designated FSB-1. This configuration achieves a 1,300 lb propellant margin with a $118,000 \mathrm{lb}$ propellant offload. Similarly, the 65 -in. stretch FSB-2 configuration achieves $7,000 \mathrm{lb}$ of increased propellant margin capability with the $118,000 \mathrm{lb}$ propellant off-load. The design attributes that were incorporated into the FSB grain designs to help match the optimum thrust profile are summarized in Figures 10,11 , and 12. Commonalities exist among all three designs from a conceptual standpoint. The propellant burn rate was adjusted to match the target web time. Significant modifications to the forward segment fin geometry were made to achieve maximum thrust and control maximum $Q$ at the bottom of the thrust bucket near fifty seconds. Center segment inhibitor heights were tailored to provide the desired performance ramping up out of the thrust bucket. Aft segment bore tapers were altered to control the thrust time trace slope during maximum vehicle accelcration. Generally speaking, these grain design approaches were applied to all three FSB concepts. The degree to which each design change was required and various other grain design subtleties varies between the concepts. It is interesting to note that there are relatively minor design feature changes required to achieve the desired correlation with the optimum profile, thereby showing there is a significant amount of flexibility in tailoring the performance characteristics of the booster to achieve optimum capability.

In the Phase A study the maximum propellant off load that was evaluated was $50,000 \mathrm{lb}$ of LOX. This off load was selected to correspond with a $\mathbf{5 . 8 5}$ mixture ratio for the SSME. The 5.85 was selected because that was the lowest mixture ratio evaluated and tested by the SSME program. From the results shown in Figure 9, it is apparent that offloads beyond $50,000 \mathrm{lb}$ are desirable to maximize abort capability. It became, therefore, important to determine whether the current 6.032 or a 5.85 mixture ratio would be most beneficial for off loads beyond $50,000 \mathrm{lb}$. A summary of the SSME performance characteristics for the two mixture ratios of interest is shown in Table 2. A comparison of the performance characteristics for both the nominal no fail

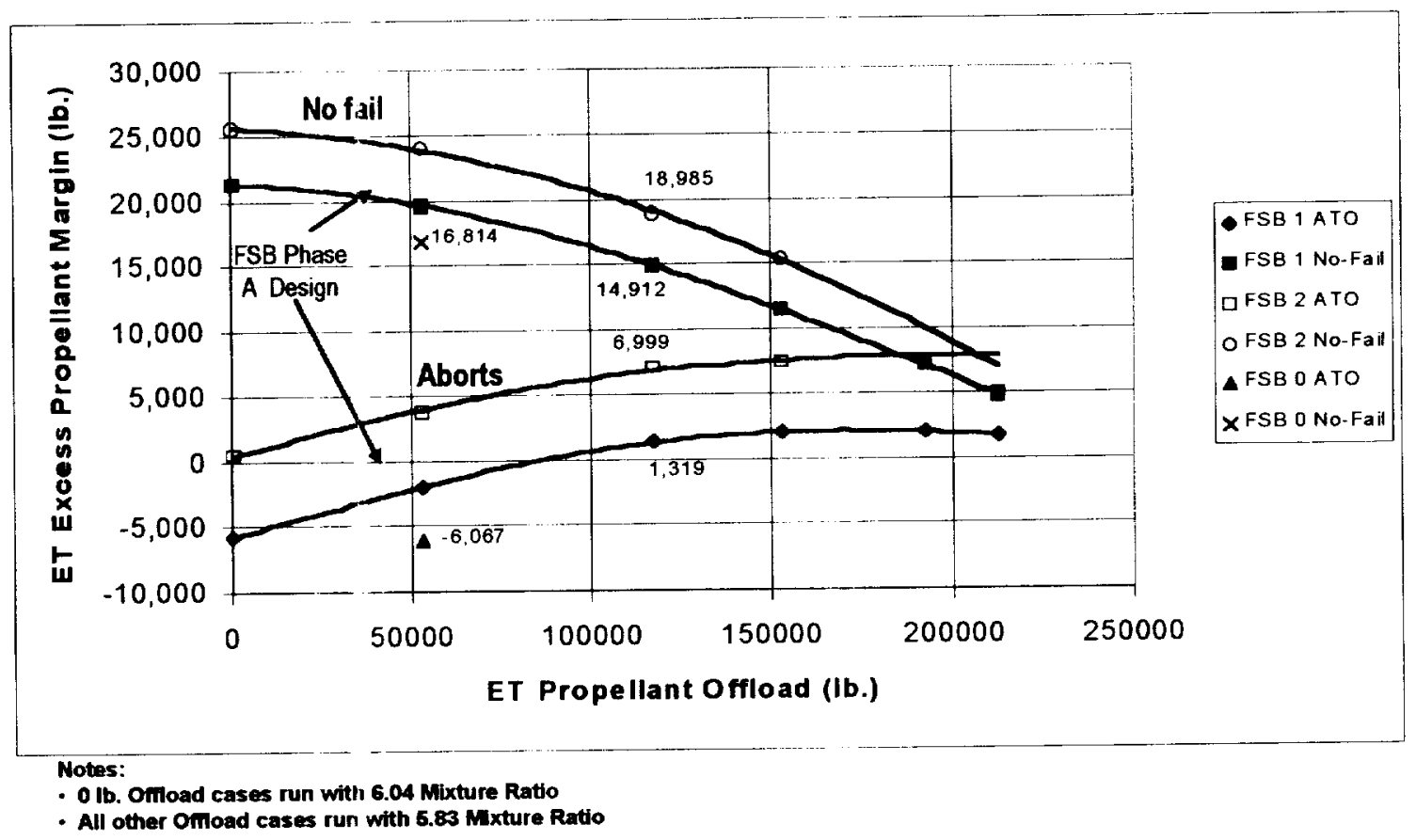

Figure 9. ET Omoad Performance 


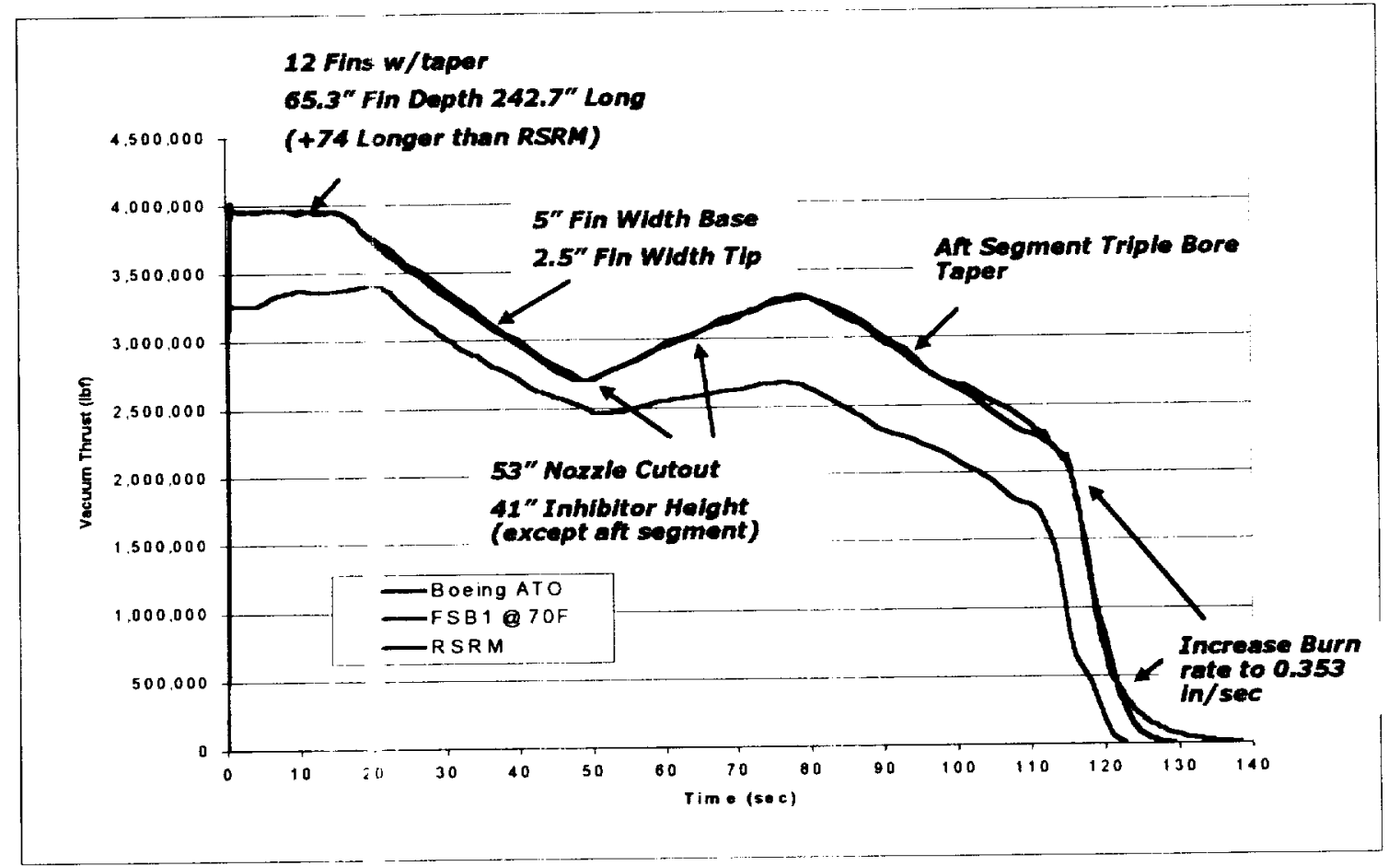

Figure 10. FSB1 Grain Design Status

mission condition and the abort condition for various propellant off-load scenarios is shown in Figure 13. Notice that for the no failure condition a mixture ratio of 5.85 provides more capability, but in both cases, there is sufficient capability to meet the station requirements. For the abort scenario, the 6.032 is slightly better than the 5.85 mixture ratio. As a result of this comparison, it was determined to continue utilizing the current 6.032

12 Fins $45^{\prime \prime}$ longer and $1^{\prime \prime}$ deepe than RSRM

Fin bore tapered from $13^{\prime \prime}$ to $16^{\prime \prime}$

Gives Maximum thrust in earty part of trace

FSB+ Thrust Profile

-FSB+ - Target

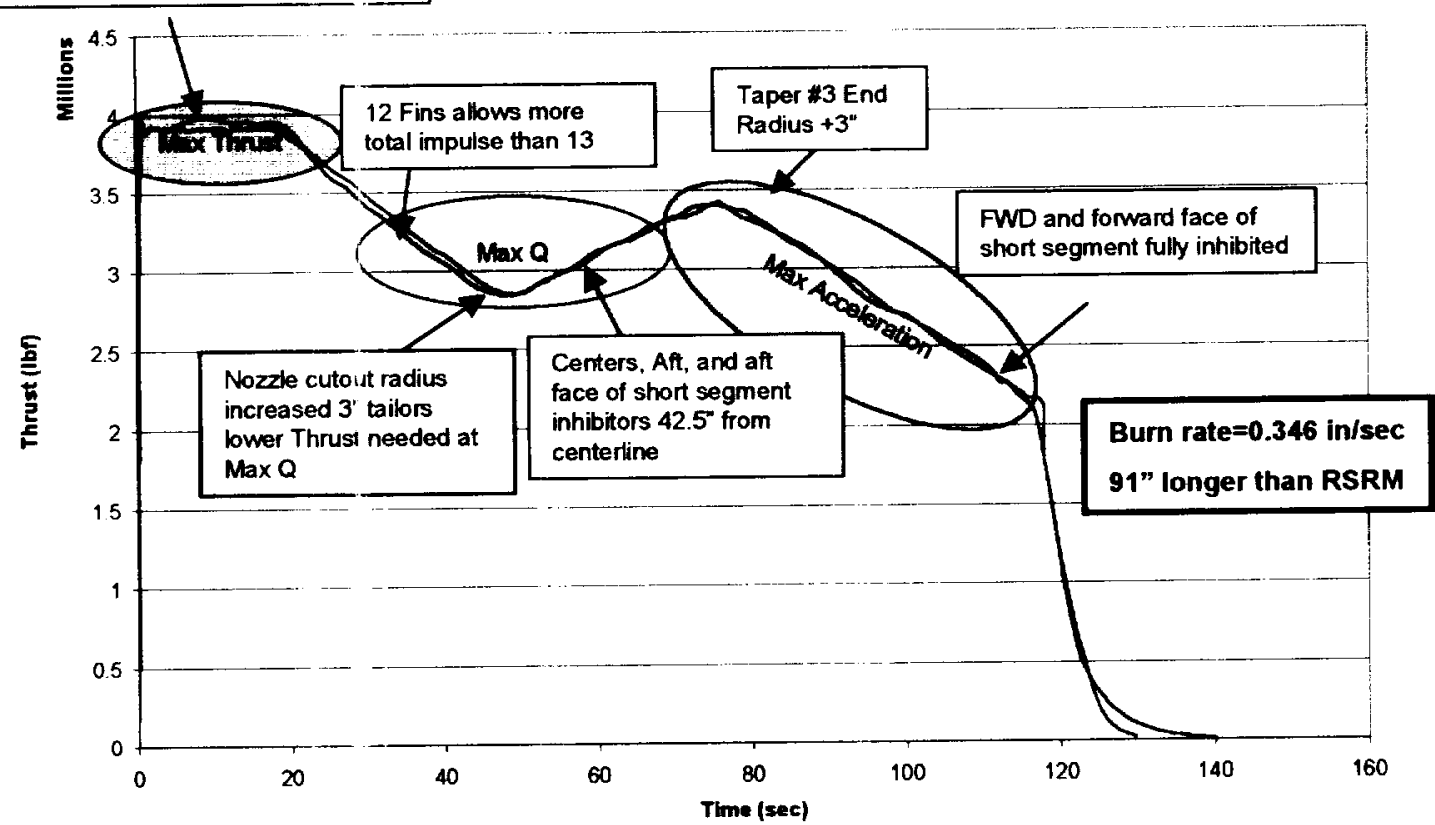

Figure 11. FSB + 65 Grain Design Status 


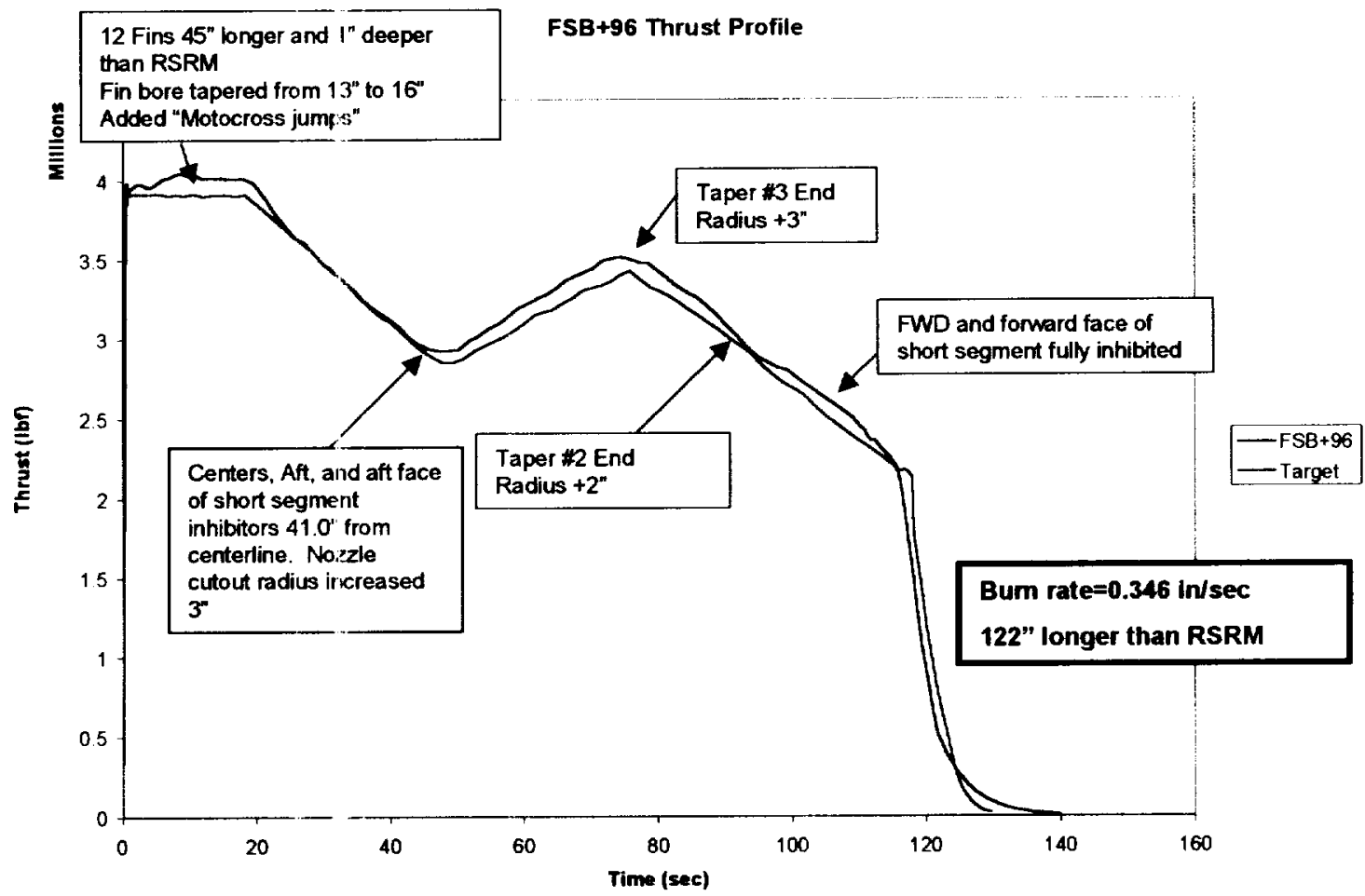

Figure 12. FSB + 96 Grain Design Status

mixture ratio as the nominal condition for future abort mode evaluations. Evaluating the difference in performance between 6.032 and 5.85 mixture ratios as a function of propellant off load is shown in Figure 14, confirming the evaluation from Figure 13 where the 6.032 mixture ratio is more optimum for the "no fail" condition.

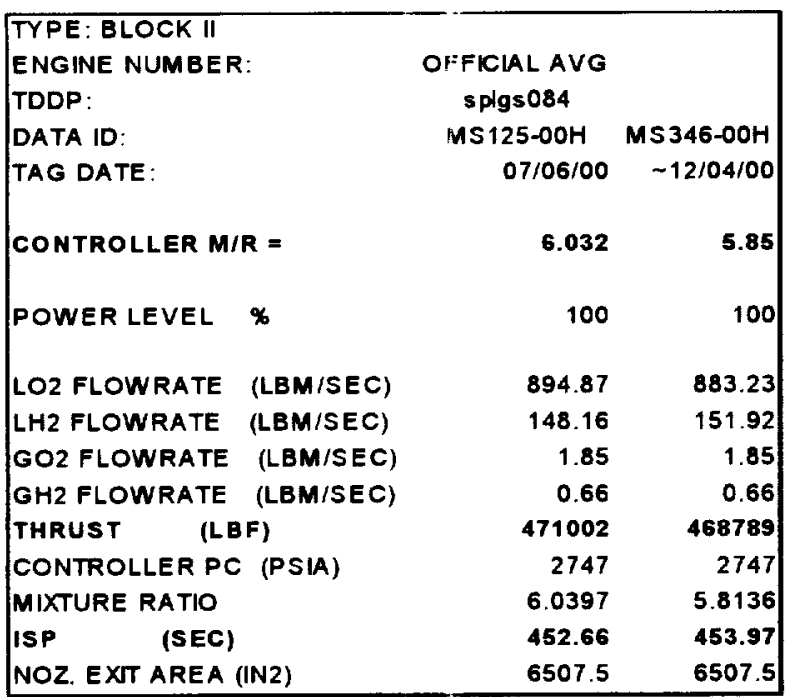

Table 2. SSME Tag Data for 6.032 and 5.85 Controller Mixture Ratios
This follow-on study confirms the capability for the FSB to enable ATO from the pad, thus eliminating the necessity for TAL and RTLS abort modes. This study also indicates the ability to not only achieve ATO, but to do so at a SSME throttle setting of 109 percent and reasonable performance margin consistent with performance changes that occur as a result of design maturation during development. These results reaffirm the importance of proceeding with more detailed evaluation and maturation of the FSB design concept. A more effective evaluation of the implications on other Shuttle elements to ensure the optimum capability identificd as a result of the optimization studies and the associated grain configurations can indeed be achieved when incorporated into the elements of the Shuttle system. 


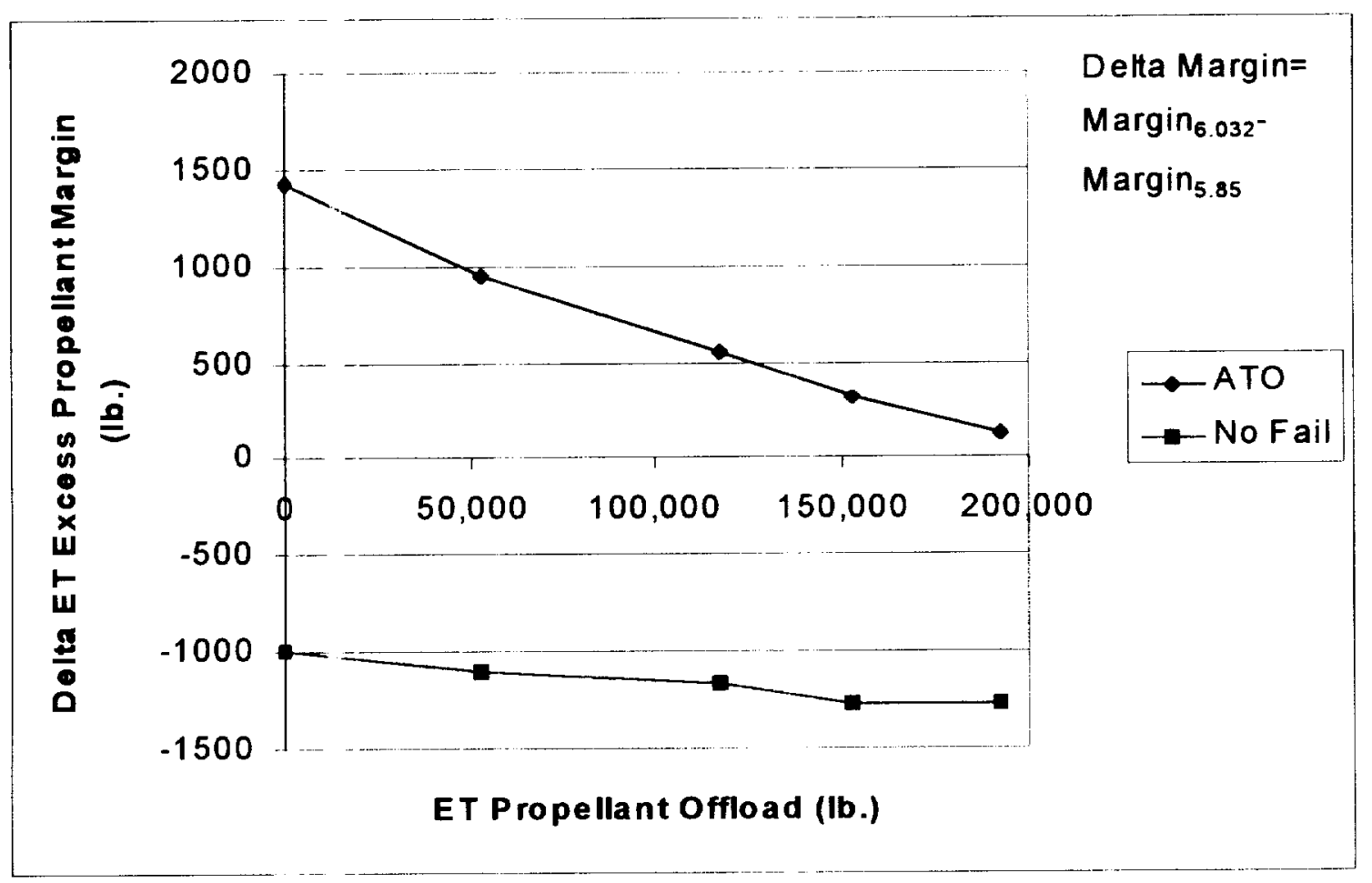

Figure 14. FSB-1 No-Fail/ATO Delta Performance with 6.032 and 5.85 SSME Controller Mixture Ratios

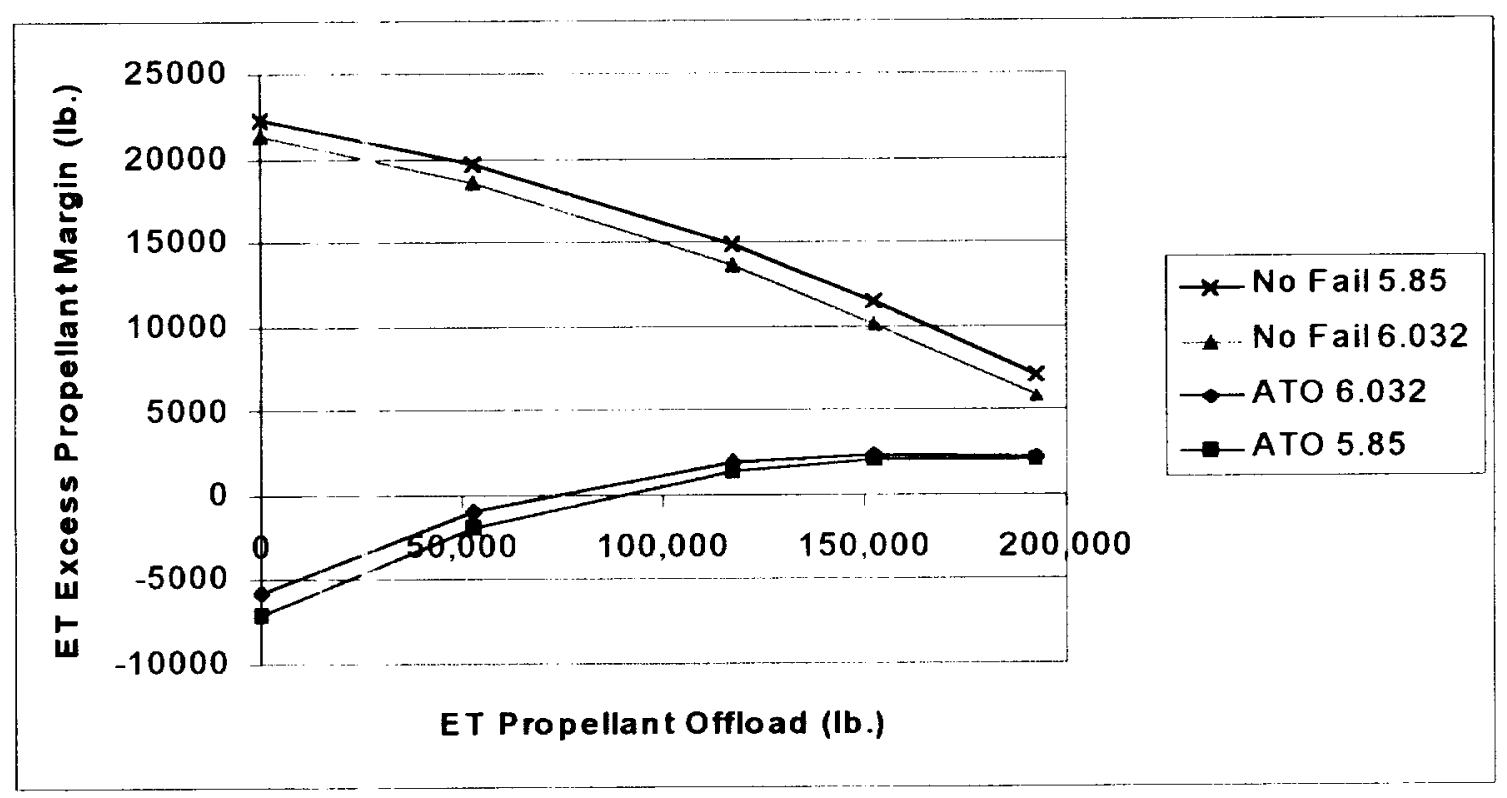

Figure 13. FSB-1 No-Fail/ATO Performance with 6.032 and 5.85 SSME Controller Mixture Ratios 


\section{REFERENCES:}

James A. Furfaro, Brian Laubacher, and Donald R. Sauvageau, "Five-Segment Booster (FSB) Phase A Study Results," presented at JANNAF Propulsion Meeting in Salt Lake City July 12-13, 2001

Donald R. Sauvageau, "Achiev ing Space Shuttle ATO Using the Five-Segment Booster (FSB)"; AlAA 20013413

Donald R. Sauvageau, Hal D. Huppi, "Shuttle Upgrade using 5-Segment Booster (FSB)." AIAA 2000-5070 